\title{
Improvement of Hierarchical Routing Algorithm in Wireless Sensor Network
}

\author{
https://doi.org/10.3991/ijoe.v15i01.9780 \\ Yinghui Yang $(\bowtie)$ \\ Henan University of Animal Husbandry and Economy, Zhengzhou, China \\ Qunting Yang \\ Civil Aviation University of China, Tianjin, China \\ Tiegang Gao \\ Nankai University, Tianjin, China \\ yinghuiyang344829@163.com
}

\begin{abstract}
To prolong the life cycle of the network and increase the effective data transmission quantity of the network, several classic network layer routing algorithms are analyzed and they are implemented with programming language, which verifies their real advantages and disadvantages. In the model of ring networking, the optimal number of cluster heads in the annular region is deduced with the goal of minimizing the average energy consumption of cluster heads in each ring, and the formation mechanism of cluster heads is proposed for the purpose of preferentially using nodes with more residual energy as cluster heads. To further optimize energy consumption performance, a dynamic self-organizing multi-hop routing selection mode is proposed. Finally, the network simulation software is applied to construct the model based on ring network, and the optimization algorithm is adopted to obtain the flow chart of actual programming and some programming methods. The results show that the proposed algorithm achieves the ultimate optimization goal, which increases the number of effective monitoring data packets.
\end{abstract}

Keywords-Wireless sensor network, hierarchical routing algorithm, cluster head

\section{$1 \quad$ Introduction}

Wireless Sensor Networks (WSN) is a task-oriented self-organizing network composed of a large number of distributed sensor nodes. Wireless sensor networks may involve microelectronics, CSOC-System-Chip, sensors, nanomaterials, wireless communications, computer networks, distributed information processing. It has broad and huge application prospects in the fields of agro-industrial control, national defense military, urban management, medical and health, environmental detection, disaster relief, and remote control in harsh conditions. 
As a new research field, wireless sensor networks put forward many challenging topics for scientists and technicians in both theoretical and practical fields, including QoS (Quality of Service) guarantee, data fusion technology, network security mechanism, positioning technology, synchronization management mechanism, network protocols and other major technologies. Because wireless sensor networks have their own unique characteristics, they are manifested in: node resources are limited (including computing power and node energy, network node topology changes frequently), addressing is data-centric (wireless sensors are more concerned with the data itself, such as events, time and region range, etc. It does not care which node the data was collected from), multi-hop routing communication, distributed characteristics of the network, etc. Therefore, the network routing protocol of wireless sensor networks has become the top priority of many research directions. It plays the role of monitoring network topology changes, establishing, maintaining and deleting inter-node routes to ensure accurate, efficient and timely delivery of information between nodes in harsh environments; however, due to the limited resources of the wireless sensor network, this determines that the research of its network protocol is different from other network protocols. Its core is to ensure maximum energy utilization efficiency and relatively optimized service quality.

In the field of wireless sensor routing protocols, great achievements have been made in recent years. Planar routing protocols, hierarchical routing protocols, energyaware routing protocols, query-based routing protocols, geographical routing protocols, and reliable routing protocols have been proposed; among them, hierarchical routing protocols are particularly prominent in terms of energy utilization efficiency and data transmission reliability. Based on the hierarchical routing protocol, an improved hierarchical routing algorithm is proposed under the condition of multi-level heterogeneous energy and large monitoring area.

Wireless sensor networks (WSNs) are composed of a large number of sensor nodes that are randomly distributed or regularly distributed in a certain monitoring area. Each node is connected by wireless multi-hop or single-hop, and can mutually sense, collect and process information for a specific object in the network coverage, and return to the observer in the manner of relaying the gateway node. Compared with the traditional network, there is a significant difference between the way to organize the network and the structure of the sensor. Wireless sensor networks include distributed sensor nodes, receivers, transmitters, the Internet, and user interface interfaces. The sensor node is the most basic component of the network. Many sensor nodes are distributed in a specific application environment. Through many network protocols, wireless network technology is used to self-organize into a network to accomplish specific tasks. Similar to other networks, wireless sensor networks adopt a layered architecture, which generally includes a physical layer, a Media Access Control (MAC) layer, a network layer, a transport layer, and an application layer. 


\section{$2 \quad$ Literature Review}

Deshmukh et al. (2016) pointed out in the research that the emergence of technology that links traditional sensors through point-to-point transmission technology and specialized link sensor controllers has led to the formation of sensor networks, often referred to as first-generation sensor networks [1]. Viattchenin (2016) pointed out that with the development of technologies such as microelectronics and System-On-Chip (SOC), sensors also have comprehensive processing capabilities for acquiring multiple information signals. The serial/parallel interface (RS-232, RS-485) is linked to the sensor controller to form a sensor network with information integration and processing capabilities [2]. Kalaiselvi et al. (2016) pointed out that the sensor can obtain a variety of information signals, and the fieldbus is connected to the sensor controller to form a local area network [3]. Verma et al. (2017) pointed out that the fourth generation of sensor networks is characterized by the use of a large number of sensors with multi-functional and multi-information signal acquisition capabilities. The connection between sensors is changed to wireless connection, forming a wireless sensor network, which is a milestone in the development of sensor networks. This greatly expands the application area of sensor networks [4].

Bongale et al. (2016) pointed out that after the middleware technology was introduced into the wireless sensor network, many research institutions began to study from different aspects. The vast majority of middleware development efforts focus on extending the life cycle of the network and how to maximize the use of the network's limited resources [5]. In addition, Solaiman et al. (2016) also pointed out that many famous universities in the United States, such as the University of California at Berkeley, have begun to explore the theoretical basis and engineering application technology of wireless sensor networks [6]. At the same time, the military departments and industries of many countries have also turned their attention to this field. Arya et al. (2016) pointed out in the study that the Natural Science Foundation of the United States established and implemented a research plan for wireless sensor networks in 2003; military units, including the US Department of Defense, have given considerable attention to wireless sensor networks and established related research projects [7]. Elhoseny et al. (2016) pointed out that the famous companies in the information industry such as Intel and Microsoft in the United States have also carried out research work in this area. The developed countries such as Japan, Germany, the United Kingdom, and Italy have also started research in this field [8].

In addition, researchers at some research institutes have conducted deeply research in this new field and have achieved fruitful research results. There is already research that is raising more questions in this area. With the overcoming of many difficulties in theory and engineering technology and the gradual decline in the price of sensor nodes, wireless sensor network will be widely used in the next 10 years. 


\section{$3 \quad$ Methodology}

\subsection{Algorithm goal}

The method of periodically electing cluster heads solves the global allocation of energy consumption well, avoiding the nodes in some areas taking too many tasks and dying in advance. On this basis, the data collection efficiency of the wireless sensor network (total energy utilization of the wireless sensor network) is better improved for the following aspects.

Self-Organizing: The idea of base station central control is proposed. The cluster head election mode is optimized by the method of base station control. However, in the large area network, the premise of this central control is that in the cluster head establishment phase, the common node needs to communicate with the base station to provide the necessary information for the base station to control the cluster head election. However, in a large-scale monitoring area, wireless communication transmitting this part of the control information will consume an extra considerable amount of energy. Therefore, in a self-organizing manner, ordinary nodes are automatically generated by certain rules in the cluster formation stage.

Layered Processing: In the case of large area and multi-level heterogeneous initial energy of nodes, there are two ways to layer: one is that in the area different from the distance from the base station, the cluster head election, data transmission, and processing rules are different; the second is that in a certain area, when the remaining energy of each node is different, the probability that the node participates in the election as a cluster head is different. The probability that a node with a large remaining energy is elected as a cluster head is large, and vice versa.

The Optimization of the Number of Cluster Heads: Since the amount of tasks undertaken by cluster heads that are different from the base station is different, if the number of cluster heads is too large, excessive data redundancy will result, which indirectly causes waste of energy; if the number of cluster heads is too small, the data processing tasks undertaken by the cluster head nodes are too large and exhausted in advance. Once a node is "dead", the entire network will become unstable and the accuracy of the collected data will be reduced. The reasons for this instability are explained in detail in later chapters. Therefore, through theoretical derivation, the optimal number of cluster heads in different types of regions is solved, and the optimal balance of energy consumption between regions is achieved. Although in the actual cluster head election, the number of cluster heads generated is not completely equal to the theoretical value, in the network stability period, the number of cluster heads generated by actual elections is basically consistent with the theoretical value, which improves the energy consumption uniformity between regions to some extent.

Multi-Hop Path Selection: The monitoring area is a large-scale environment. Therefore, the cluster heads in each hierarchical area combine the information data of the cluster members and send them to the aggregation node with multi-hop routing. When selecting a route, the cluster head in the delimited candidate area is used as the alternate forwarding node. The cluster head node performs the priority mode when selecting the forwarding node. The appropriate forwarding node is selected in a 
relatively optimal manner to avoid a cluster head as a plurality of other cluster head forwarding nodes when the cluster head is selected for forwarding, resulting in excessive consumption of energy of a certain node.

\subsection{The structure of wireless sensor network node}

In the sensor network, the sensor node plays a vital role and is the basic platform that constitutes the wireless sensor network. It completes the data collection and transmission in the monitoring area, and the power module is also responsible for the node drive. Therefore, the advancement of node technology is closely related to the development of wireless sensor networks, which directly determines the performance of the entire network. The basic structure of the sensor node is shown in Figure 1.

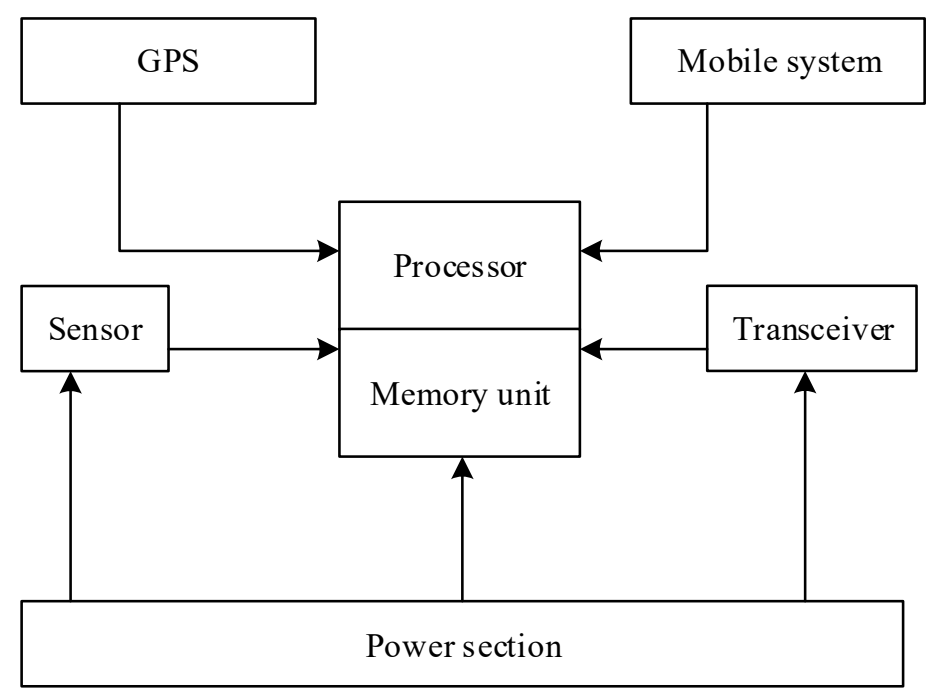

Fig. 1. The structure of the sensor node

Sensing Unit: It consists of a sensor and an analog-to-digital conversion function module (A/D), which is responsible for information acquisition and data conversion. The type is determined by the form of the monitored physical signal, such as a platinum resistance sensor for temperature monitoring and a capacitive sensor for pressure sensing, etc.

Processing Unit: It consists of an embedded system, including CPU, memory, embedded operating system, etc. The data processing unit is responsible for the internal operations of the sensor node, such as running a high-level network protocol, processing self-acquired data, and forwarding data that needs to be routed from other nodes. It usually uses a low-power embedded microcontroller.

Communication Unit: It consists of a wireless communication module. The module is responsible for communication with other sensor nodes and is responsible for data transmission and reception. Its main components include short-range wireless 
communication circuits operating in a low-power range, and communication protocols responsible for response (mainly MAC protocols).

Power: It provides the sensor with the energy needed for normal operation and is also an element of the node's life cycle. The characteristics of the wireless sensor network determine that the sensor can only use AA-type alkaline batteries or scandium batteries at this stage, but researchers have explored ways to obtain energy from the surrounding environment and convert it into electrical energy.

In addition, nodes in some wireless sensor networks may also have other functional units, such as positioning systems, mobile systems, etc. Positioning systems are primarily used in applications that require detailed geographic location for monitoring data, while mobile systems enable nodes to have the ability to change locations.

The design of the sensor nodes is different in different applications. Among the various node design schemes that have emerged, the implementation principles of each scheme are basically similar, except that different microprocessors and wireless communication protocols are used, such as custom protocol, 802.11 protocol, ZigBee protocol, Bluetooth protocol, and USB communication ways, etc. The basic principle is to use sensors that are as sensitive as possible, devices with the lowest power consumption, signal processing methods with the highest efficiency, and power supplies with the longest possible endurance. However, sensor nodes often fail or are discarded due to power supply energy. Power supply energy constraints are a serious problem that hinders wireless sensor network applications. The energy consuming module includes a sensor module, a processor module, and a wireless communication module, wherein the transceiver consumes a large portion of the total energy consumed by the wireless communication module.

\subsection{Algorithm improvement based on ring networking}

The sensor nodes in each ring start the cluster head election according to the election probability in the ring. Once the sensor node in a ring becomes a cluster head node by means of election, the node broadcasts a message CH_ADV_MSG packet, and the packet contains specific location information of the node. When other common nodes receive multiple CH_ADV_MSG type data packets, the cluster head closest to itself is determined according to the strength of the message signal, and the JOIN_MSG data packet is sent to it. Considering that the cluster head is transmitted by broadcast, the factors of access collision and interference are not excluded, and thus a small number of sensor nodes do not receive any $\mathrm{CH}$ _ADV_MSG message. The solution in the LEAC $\mathrm{H}$ algorithm is to let such nodes send data directly to the gateway node, but in the case of a large monitoring area, this method is impossible due to the limited communication range capability of a single sensor node. Therefore, after the cluster head broadcast ends, the node that has not received any CH_ADV_MS G message will actively broadcast the CH_REQ_MSG message, and the message contains its own ID. The corresponding cluster head that received the packet message sends a CH_ACK_MSG message to it unicast. Through this process, the reliable establishment of the cluster is guaranteed. 
In a data collection cycle, the cluster member node collects information of interest to the user in the monitoring area and aggregates it and sends it to the cluster head. The cluster head node performs a data fusion policy to process information from the cluster members. Because the information correlation between the cluster and the cluster has been small, it is very difficult to perform data fusion. Therefore, the forwarding cluster head node does not perform any processing on the source node from the previous hop, and only performs the forwarding operation to the next hop. Therefore, in a wireless sensor network based on a ring networking and performing a multi-hop path, since the cluster head closer to the gateway node has a greater forwarding pressure than the cluster head node in other areas, it is easy to decay prematurely. In order to overcome this problem, the energy consumption between cluster heads of different rings is balanced by controlling clusters of different sizes in different loops. To sum it up, the energy of its cluster head in intra-cluster communication and data aggregation is reduced, so that the cluster head consumes less energy in intra-cluster communication and data aggregation to compensate for the energy consumption caused by forwarding the data packets of the outer ring cluster head; the larger the ring that is farther from the gateway node, the larger the size of the cluster, so that its energy consumption in intra-cluster communication and data fusion is increased to offset the energy saved by the less forwarded data. The size of the average cluster in a ring is controlled by the number of cluster heads of the ring, and the optimal cluster head number of a ring is obtained, and the optimal cluster head election probability of the ring is determined.

\subsection{Algorithm design implementation steps}

The network starts: When all nodes are randomly deployed (including gateway nodes), the entire network is formed. At this time, the gateway node needs to send a startup information, that is, a Sink_MSG data packet, to all nodes in the network. The main purpose of this packet is to obtain all the necessary information including all nodes. The format of the Sink_MSG packet is shown below:

\begin{tabular}{|l|l|l|l|}
\hline SrcAdr & DestAdr & Type & Staus \\
\hline
\end{tabular}

Fig. 2. Startup message Sink_MSG

As shown in Figure 2, where SrcAdr represents the source node of the message and De stAdr represents the destination node of the message, Type indicates the type of message: 0 (start information mode), 1 (cluster head election mode), 2 (route discovery assistance mode), 3 (data transmission mode), Staus indicates the state of the node: 0 (start), 1 (ready), 2 (death), 3 (warning).

It can be concluded that the network starts, and the source node SrcAdr of the information sent by the gateway node represents the gateway node. DestAdr represents all common nodes, the value of Type is " 0 ", which means that all nodes send their own necessary information to the gateway node. The value of Staus should 
be " 0 ", indicating that the gateway node is ready. And sink_Message is broadcasted in a flooded manner (Each node that receives the packet for the first time will broadcast to its own communication radius once, and the packet can reach each node in the area).

The formation of clusters and cluster heads: After all nodes receive the data packet Sink MSG from the gateway node, each node starts to send the data packet Node_Message to the gateway node in the same manner as flooding. The specific form of the data packet is shown in Figure 3:

\begin{tabular}{|l|l|l|l|l|l|l|}
\hline SrcAdr & DestAdr & Type & Staus & $\mathrm{X}$ & $\mathrm{Y}$ & $\mathrm{ID}$ \\
\hline
\end{tabular}

Fig. 3. Node_Message

As shown in Figure 3, SrcAdr should indicate that the source node is a normal node, and De stAdr represents a gateway node; the state of the Type shall be the basic information of the sending ordinary node itself, including the position coordinates $\mathrm{X}$, $\mathrm{Y}$, and the flag information D number of the node.

When the gateway receives all the information sent by the ordinary node, it forms a data information table. The schematic diagram of the information table is shown in Figure 4:

\begin{tabular}{|c|c|c|}
\hline ID & $X$ & $Y$ \\
\hline 4 & 102.32 & 94.57 \\
\hline 270 & 212.68 & 75.89 \\
\hline 9 & 91.73 & 354.68 \\
\hline 120 & 176.58 & 109.83 \\
\hline 23 & 297.13 & 50.68 \\
\hline
\end{tabular}

Fig. 4. Data distribution map of gateway nodes

\section{$4 \quad$ Result}

\subsection{Startup method of algorithm}

Questions about the routing table: Any node has its own routing table, and its role is: after the cluster head completes the data collection and data fusion of its cluster members, it manages the lookup of the forwarding cluster head node in the next ring close to the gateway node and causes the D number of the cluster head node responsible for forwarding to appear in the routing table of the current source cluster 
head node. It can be concluded that the routing table only plays a role after the ordinary node becomes the cluster head node, and the routing table of the ordinary node is in the dormant state.

The role of the gateway node: In wireless sensor networks, gateway nodes play a vital role. At the same time, the general energy of the gateway node is much larger than that of the ordinary node. It carries the control of the operation of the entire network, calculates the corresponding reference information, receives the data packets sent by the cluster, and performs data fusion different from the cluster nodes (the fusion degree is higher than cluster node). Therefore, there is no need to consider the problem of energy optimization here, that is, the energy of the gateway node is assumed to be large enough. Specifically, the function of the gateway node is to timely release global information to each common node and provide necessary reference information for the election of the cluster head. Through the interaction with the common node at the beginning of the cycle, the optimal number of cluster heads in each ring is calculated, and the threshold value in any ring when electing a cluster head is derived. When the forwarding node is selected from the cluster head away from the gateway node, the necessary reference information to be calculated is provided to assist in discovering the optimized route. The gateway node receives information from clusters in all rings and makes a high degree of data fusion. It publishes task information to the normal node and monitors the amount of data that needs to be monitored.

\subsection{Simulation scene settings}

Any design or improved protocol algorithm requires scientific and objective evaluation of the indicators of the wireless sensor network under any environmental conditions, so as to derive the advantages and disadvantages of the improved or designed algorithm as an important reference data for its application in the actual scene. But building a real wireless sensor network is limited by many factors, especially the performance of the algorithm in different environments needs to build a very different number of networks, it is difficult to get results through experiments. Therefore, network simulation solves this problem well and provides a good platform for network performance. The wireless sensor network is built simultaneously by NS2 and Matlab software, and the proposed algorithm is evaluated for different indicators, and the final conclusion is made.

Because the proposed algorithm is directed to two specific conditions of multilevel heterogeneous of energy and medium-scale monitoring area. The specific environment of energy multi-level isomerization and medium-to-large-scale monitoring range is closer to the actual application, as the monitoring environment is harsh, the complexity of the algorithm becomes correspondingly complex.

In order to make the performance of the algorithm more objective and comprehensive, the simulated scene should include large areas, medium areas and small areas, and the energy types are divided into isomorphism and heterogeneity. The specific simulation scenario is shown in Table 1: 
Table 1. Simulation scene parameter table

\begin{tabular}{|c|c|c|c|}
\hline Scale & Nodes & Energy & Rings \\
\hline $100 \mathrm{~m}$ & 100 & $1 \mathrm{~J}$ & 1 \\
\hline $100 \mathrm{~m}$ & 100 & $1 \mathrm{~J}-2 \mathrm{~J}$ & 2 \\
\hline $160 \mathrm{~m}$ & 200 & $2 \mathrm{~J}$ & 2 \\
\hline $160 \mathrm{~m}$ & 200 & $5 \mathrm{~J}$ & 3 \\
\hline $240 \mathrm{~m}$ & 1000 & $5 \mathrm{~J}-7 \mathrm{~J}$ & 3 \\
\hline $240 \mathrm{~m}$ & 1000 & $8 \mathrm{~J}$ & 4 \\
\hline $720 \mathrm{~m}$ & 8000 & $8 \mathrm{~J}-10 \mathrm{~J}$ & 4 \\
\hline $720 \mathrm{~m}$ & 8000 & $12 \mathrm{~J}$ & \\
\hline
\end{tabular}

\subsection{Simulation results and analysis}

One of the great features of the routing algorithm for wireless sensor networks is that different algorithms are required to match depending on the application. Because various routing algorithms have their own unique characteristics, specific application scenarios and scope of application. Similarly, the proposed algorithm also has its advantages and disadvantages and scope of application.

Transmit data packets: the scene is $100 \mathrm{~m}$ in size, 100 in number of nodes, and no split-ring system. Figure 5 shows the number of packets received from the cluster head in the isomorphic case, and Figure 6 shows the number of packets received from the cluster head in the heterogeneous case.

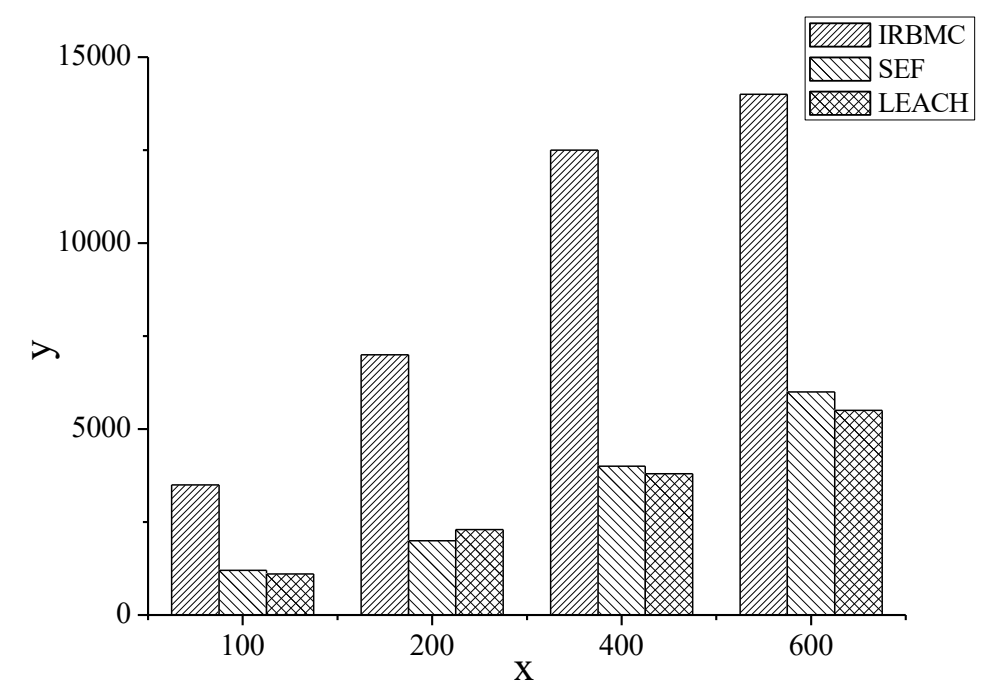

Fig. 5. Number of packets received from the cluster head (isomorphic) 


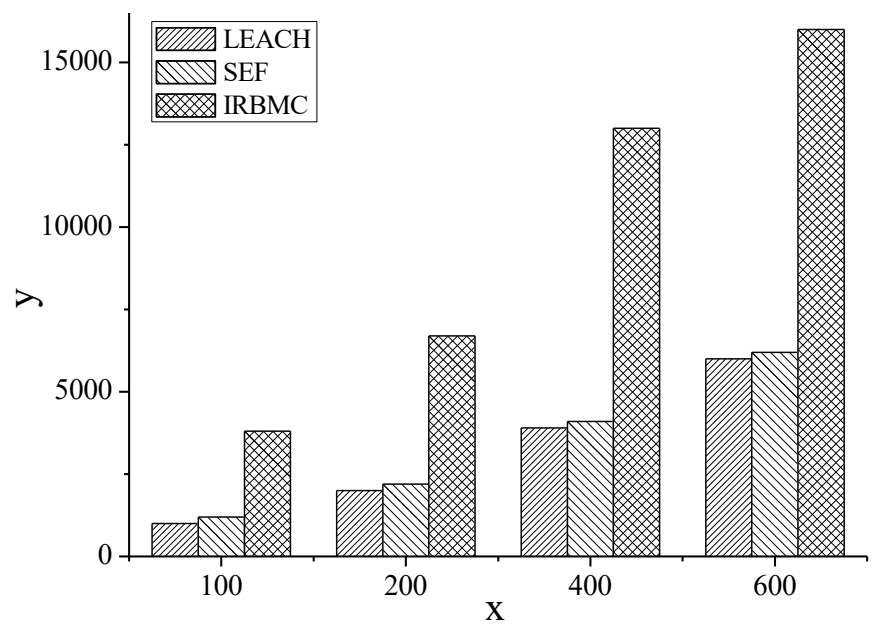

Fig. 6. Number of packets received from the cluster head (heterogeneous)

The scenario is $160 \mathrm{~m}$ in size, the total number of nodes is 200 , and the number of rings is 2 . Figure 7 shows the number of packets received from the cluster head in the isomorphic case, and Figure 8 shows the number of packets received from the cluster head in the heterogeneous case.

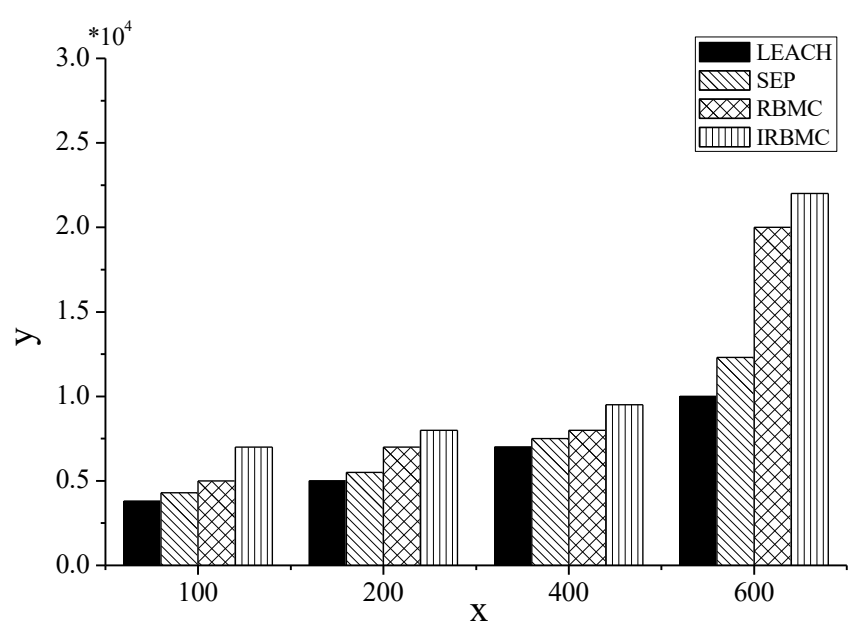

Fig. 7. Number of packets received from the cluster head (isomorphic) 


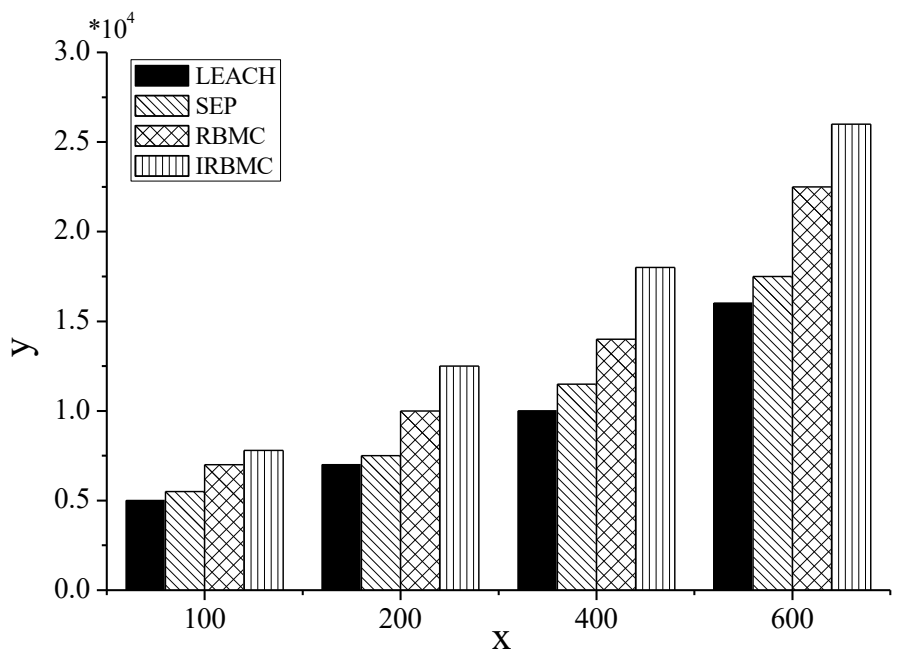

Fig. 8. Number of packets received from the cluster head (heterogeneous)

Figures 5 through 8 depict the relationship between the number of simulated rings and the number of received packets in four simulation environments. The environment examined is a circular area with a radius of $100 \mathrm{~m}$. Compared with SEP and LEACH, IRBMC has a significantly larger number of data packets when the number of simulation rings is $100,200,400,600$. This is because, when the monitoring area is small, the data packets used in the reference information in the IRBMC are greatly increased. The transmission of this part of the data packet will undoubtedly greatly increase the energy burden of the nodes, but these data packets are not effective monitoring information. Therefore, it is concluded that in the small area monitoring range, the transmission of reference information data packets is completely unnecessary, and the SEP and LEACH algorithms are more in line with the application requirements. In the large area monitoring range, due to the limitation of the communication distance of common nodes, a multi-hop routing strategy is adopted. The acquisition of reference information is only a large number in the initial stage of system operation. When the monitoring data transmission starts, the number of additional control data packets is small and stable. Therefore, due to the adoption of the proposed optimization strategy, at this time, as the number of simulation rounds increases, the number of data packets received by the IRBMC and the RBMC gateway node is much larger than that of the SEP, LEACH; moreover, the proportion of effective monitoring information packets also increases. It can be concluded that after the radius is greater than $240 \mathrm{~m}$, the number of data packets received by the IRBMC gateway node is always greater than the RBMC as the number of simulation rounds increases. Therefore, based on EZBMC, the IRBMC algorithm achieves the ultimate optimization goal, which increases the number of effective monitoring data packets. 


\section{Conclusion}

A ring-based networking model is proposed, which includes the ring network mode, energy consumption model, energy multi-level heterogeneous representation and the objectives to be achieved. A detailed theoretical derivation process analysis is given. In the ring networking mode, the calculation method of the optimal number of cluster heads in the sub-ring region is derived by balancing the minimization of the average energy consumption of the cluster heads in each ring. In order to preferentially use the node with more residual energy as the cluster head, the formation mechanism of the cluster head is proposed. In order to further optimize the energy consumption performance, a dynamic self-organizing multi-hop routing method is proposed. Finally, the proposed ring network-based model is constructed and the optimization algorithm is proposed with the network simulation software. The actual programming flow chart and some programming methods are given. Under the four indicators of life cycle, energy consumption rate, number of cluster heads in each round, and data transmission volume, the final reaction performance data is obtained, and a detailed conclusive analysis is made.

\section{Reference}

[1] Deshmukh, M., \& Gawali, D. (2016). Optimal probabilistic cluster head selection for energy efficiency in wsn. 2 (12): 1249-1256.

[2] Viattchenin, D. A. (2016). Heuristic possibilistic clustering for detecting optimal number of elements in fuzzy clusters. Foundations of Computing \& Decision Sciences, 41(1): 45-76. https://doi.org/10.1515/fcds-2016-0003

[3] Kalaiselvi, T., \& Murugan, T. S. (2016). An improved load balanced connection aware clustering hierarchy protocol for military application in mobile ad hoc network. Research Journal of Applied Sciences Engineering \& Technology, 12(6): 658-667. https://doi.org/10.19026/rjaset.12.2714

[4] Verma, A., Rashid, T., Gautam, P. R., Kumar, S., \& Kumar, A. (2017). Fuzzy based stable clustering protocol forheterogeneous wireless sensor networks. International Journal of Engineering \& Technology, 9(4): 2854-2860. https://doi.org/10.21817 /ijet/2017/v9i4/170904046

[5] Bongale, A. M., \& Nirmala, C. R. (2016). Eoichd: a routing scheme for wireless sensor network based on energy and optimal inter cluster head distance. International Journal of Applied Engineering Research, 11(11): 7256-7266.

[6] Solaiman, B., \& Sheta, A. F. (2016). Evolving a clustering algorithm for wireless sensor network using particle swarm optimisation. 2(1): 43.

[7] Arya, N., \& Suryawanshi, V. (2016). Distance based cluster head selection model for optimum number of clusters in wsn. International Journal of Computer Applications, 156(1): 58-63.

[8] Elhoseny, M., Elminir, H., Riad, A., \& Yuan, X. (2016). A secure data routing schema for wsn using elliptic curve cryptography and homomorphic encryption. Journal of King Saud University - Computer and Information Sciences, 28(3): 262275 . 


\section{$7 \quad$ Authors}

Yinghui Yang is work in School of Information Engineering, Henan University of Animal Husbandry and Economy, Zhengzhou Henan, 450045, China, His research interests include Optimization Algorithm.

Qunting Yang is work in College of Air Traffic Management, Civil Aviation University of China, His research interests include wireless sensor network.

Tiegang Gao is work in College of Software, Nankai University ,Tianjin 300071 ,China, His research interests include hierarchical routing algorithm

Article submitted 24 October 2018. Resubmitted 30 November 2018. Final acceptance 12 December 2018. Final version published as submitted by the authors. 